\title{
Relación entre factores sociodemográficos y clínicos con la discapacidad visual en pacientes atendidos en dos instituciones oftalmológicas de la ciudad de Medellín durante el 2015
}

\section{Relationship between sociodemographic and clinical factors with visual impairment in patients attended at two ophthalmological institutions of the city of Medellín during 2015}

\author{
Aníbal J. Cabrales-López ${ }^{1,2,3}$, John A. Isaza-Arias ${ }^{1,2,3}$, Laura Quintero-Gutiérrez ${ }^{1,2,3}$, \\ Christian Ramírez-Rodríguez ${ }^{1,2,3}$, Valentina Serna-Loaiza ${ }^{1,2,3}, Y_{\text {Y }}$ dy Aguilar ${ }^{1}$ y Juan C. Suárez-Escudero ${ }^{1,2 *}$ \\ ${ }^{1}$ Facultad de Medicina; ${ }^{2}$ Línea de investigación en discapacidad, grupo de investigación Salud Pública; ${ }^{3}$ Semillero de la Facultad de Medicina. \\ Escuela de Ciencias de la Salud (ECS), Universidad Pontificia Bolivariana (UPB), Medellín, Colombia
}

\begin{abstract}
Resumen
La discapacidad visual representa un reto significativo en la calidad de vida, generando aumento en los años de vida ajustados por discapacidad. Conocer los factores de riesgo asociados permite construir acciones hacia esta población, ya que gran parte de su etiología es prevenible, y las limitaciones y restricciones en el funcionamiento se pueden mejorar con un diagnóstico precoz y rehabilitación funcional-integral. Es importante conocer los riesgos tanto sociales como clínicos a los que se ve expuesta esta población. Objetivo: Determinar la relación entre factores sociodemográficos y clínicos con la discapacidad visual en pacientes atendidos en dos instituciones oftalmológicas de Medellín durante el año 2015. Metodología: Estudio transversal, analítico en una población de 180 pacientes con alguna deficiencia visual permanente, atendidos en dos instituciones especializadas. Resultados: En el grupo de pacientes con discapacidad visual, el promedio de edad fue cercano a los 50 años, predominó el sexo femenino (55.6\%), bajo nivel de escolaridad (50.9\%), la no actividad laboral (67.9\%), consumo de alcohol (23.6\%), tabaco (15\%) y sustancias psicoactivas (4.71\%); el 67.9\% tuvo al menos una comorbilidad oftalmológica y no oftalmológica, destacándose la diabetes mellitus (7.5\%) y la hipertensión arterial (33.9\%). Conclusión: En los pacientes con discapacidad visual se encontraron mayores porcentajes de las variables mencionadas respecto a pacientes con deficiencia visual, pero sin criterio de discapacidad visual, lo que sugiere relación de estas con la discapacidad, ya sea como factor de riesgo o como desenlace, por lo que sería preciso realizar estudios con mayor población para poder encontrar significancia estadística.
\end{abstract}

Palabras clave: Personas con discapacidad. Personas con daño visual. Baja visión. Ceguera. Evaluación de la discapacidad.

\section{Abstract}

Visual impairment represents a significant challenge on quality of life, generating an increase in the years of life adjusted for disability. Awareness on the associated risk factors allows taking actions to benefit this population, since most causes are 
preventable, and limitations and restrictions on functioning can be improved with an early diagnosis and functional-comprehensive rehabilitation; it is important to know the social and clinical risks to which this population is exposed. Objective: To determine the relationship between sociodemographic and clinical factors with visual impairment in patients treated at two ophthalmological institutions in Medellín during 2015. Methodology: Cross-sectional, analytical study in a population of 180 patients with some degree of permanent visual impairment, attended at two specialized institutions. Results: In the group of patients with visual impairment, the average age was close to 50 years and the following characteristics predominated: female sex (55.6\%), low level of education (50.9\%), unemployment $(67.9 \%)$, alcohol $(23.6 \%)$, tobacco (15\%) and psychoactive substance (4.71\%) use; $67.9 \%$ had at least one ocular and non-ocular comorbidity, especially diabetes mellitus (7.5\%) and arterial hypertension (33.9\%). Conclusion: In patients with visual impairment, higher percentages of the mentioned variables were observed compared to patients with visual deficiency but without criteria for visual impairment, suggesting a relationship between these with disability, either as a risk factor or as an outcome. Thus, more studies with a larger population are needed to evaluate statistical significance.

Key words: Persons with disabilities. Visually impaired persons. Low vision. Blindness. Disability assessment.

\section{Introducción}

La discapacidad actualmente se aborda como un asunto intersectorial, centrada en el funcionamiento humano, el cual abarca las funciones y estructuras corporales, la realización de actividades y la participación. En otros términos, la discapacidad es un fenómeno universal que pone en diálogo lo corporal, lo individual y lo social' ${ }^{1}$. En el contexto de los órganos sensoriales, la discapacidad visual se define como aquellas alteraciones funcionales o estructurales que afectan la percepción de imágenes de manera parcial o total, ya sea por baja agudeza visual, campo visual disminuido o por incapacidad para distinguir la intensidad luminosa. De esta forma, el término discapacidad visual abarca la baja visión y la ceguera ${ }^{2}$.

En el mundo, la prevalencia de personas con alguna discapacidad es del $15 \%$ (cerca de mil millones de personas) $)^{3}$, y una de las deficiencias con mayor prevalencia dentro de las cifras mundiales es la discapacidad visual $^{4}$. Una revisión sistemática y metaanálisis sobre discapacidad visual estimó que en el mundo hay cerca de 36 millones de personas invidentes y 217 millones con baja visión ${ }^{5}$. Puesto que cerca del $80 \%$ de todas las deficiencias visuales pueden prevenirse, es importante investigar cuáles son las características clínicas y sociodemográficas en esta población $n^{6,7}$. Una vez certificada la discapacidad visual es importante conocer los riesgos tanto sociales como clínicos a los que se ve expuesta esta población con el fin de lograr un mejor pronóstico por medio de un enfoque integral, ya que las limitaciones y restricciones en el funcionamiento se pueden mejorar o aminorar por medio de procesos de rehabilitación funcional y acciones de accesibilidad.

En la actualidad, la etiología de las deficiencias visuales permanentes está relacionada con la edad mayor a 50 años y la presencia de enfermedades oculares crónicas como la degeneración macular asociada a la edad (DMAE), glaucoma y retinopatía diabética. Aún los errores refractivos no corregidos y las cataratas no operadas continúan siendo las causas más importantes de baja visión y ceguera, potencialmente evitables, desde una mirada de salud pública ${ }^{6}$.

En Colombia, si bien hay algunos estudios descriptivos de caracterización de población con discapacidad visual, como el que se realizó en dos instituciones de Medellín entre los años 2014 y $2015^{8}$ y el de la Fundación Saldarriaga Concha del año $2010^{9}$, no hay estudios analíticos que tengan como objetivo estudiar y establecer asociaciones entre los principales factores sociodemográficos y clínicos con la discapacidad visual, como lo son la edad mayor de 50 años, sexo femenino, bajo nivel educativo, tabaquismo, consumo de alcohol y sustancias psicoactivas, comorbilidades oftalmológicas y no oftalmológicas.

En otras partes del mundo, existen estudios analíticos sobre factores asociados a la discapacidad visual. Un estudio coreano reportó que la discapacidad visual estuvo significativamente asociada con la edad, en donde la prevalencia aumentó rápidamente a partir de los 70 años (odds ratio [OR]: 1.92; intervalo de confianza del 95\% [IC 95\%]:1.45-2.53); las mujeres presentaron un riesgo $55 \%$ mayor que los hombres para presentar discapacidad visual (OR: 1.55; IC 95\%: 1.28-189), y las personas con un nivel educativo inferior presentaron mayor riesgo de discapacidad visual que los que tuvieron educación superior (OR: 1.51; IC 95\%: 1.15-1.99) ${ }^{10}$.

Un estudio realizado en el año 2011 encontró una relación causal significativa entre el tabaquismo y la catarata nuclear, oftalmopatía de Graves, irritación e isquemia ocular, y una relación sugestiva entre el tabaquismo y la DMAE ${ }^{11}$. Junto al tabaco, el consumo de alcohol es 
otro factor que puede provocar daño ocular, pues puede generar un daño a nivel de los fotoprotectores de la retina, y evitar que se regeneren y causar daños irreversibles $^{12}$. Adicionalmente, se ha descrito una pobre relación entre el consumo de sustancias psicoactivas, principalmente inhalantes, y el desarrollo de enfermedades oftalmológicas, en su mayoría retinopatías ${ }^{13}$.

Referente al nivel educativo, se ha encontrado que la población con discapacidad visual tiene menor probabilidad de ingresar en la escuela, permanecer en ella y superar los cursos $^{3}$. Con relación a las comorbilidades oftalmológicas, cuando la discapacidad visual se acompaña de otro defecto, sea refractivo u otra comorbilidad oftalmológica, el pronóstico es más pobre, y con relación a las comorbilidades no oftalmológicas, la hipertensión arterial (HTA) y la diabetes mellitus (DM) son las patologías que más repercusión tienen a nivel ocular, las cuales se han relacionado con enfermedades oculares crónicas ${ }^{11}$.

Por lo tanto, aunque existan trabajos anteriores que relacionen algunos factores sociodemográficos con enfermedades oculares crónicas o discapacidad visual, realizados en otros países, este estudio es el primer trabajo de carácter analítico que estudió pacientes colombianos con discapacidad visual, lo cual es importante, puesto que Colombia es un país en vía de desarrollo, con falencias en promoción y prevención en salud, que cuenta con mayor prevalencia de muchos de los factores clínicos y sociodemográficos expuestos, con varias barreras en la atención en salud e inclusión de personas con discapacidad: una de cada tres personas con discapacidad se encuentra excluida de los servicios de salud, el $34.18 \%$ excluido de su derecho a la educación, la tasa de analfabetismo es del $35.9 \%$, y el $92.4 \%$ está en edad de trabajar, pero solo el $14.9 \%$ trabaja $^{9}$.

\section{Objetivo}

Determinar cuál es la relación entre algunos factores sociodemográficos y clínicos con la discapacidad visual en pacientes atendidos en dos instituciones de oftalmología de la ciudad de Medellín durante el año 2015.

\section{Metodología}

\section{Tipo de estudio y pacientes}

Estudio transversal, analítico, en una población de 180 pacientes con alguna deficiencia visual permanente, atendidos en dos instituciones especializadas de la ciudad de Medellín durante el segundo semestre del año 2015 que cumplieron los criterios de elegibilidad.

\section{Criterios de elegibilidad}

Los criterios de inclusión fueron: pacientes captados previamente en el marco del proyecto «Certificación de discapacidad sensorial visual: Aplicación de la Clasificación Internacional del Funcionamiento, Salud y Discapacidad (CIF-2001) en una población de 180 personas con déficit visual» (estudio realizado por la línea de investigación en discapacidad, del grupo de investigación en salud pública de la escuela de ciencias de la salud de la Universidad Pontificia Bolivariana, sede Medellín/Colombia), de cualquier edad, de ambos sexos, con presencia de algún déficit visual certificado 0 déficit sensorial visual secundario a alguna enfermedad crónica o trauma ocular. Los pacientes fueron captados de centros oftalmológicos de referencia de la ciudad de Medellín que atienden población del régimen contributivo y subsidiado.

Los criterios para identificar y validar la presencia o no de discapacidad visual se basaron en la agudeza visual mejor corregida, en el único o mejor ojo, después del mejor tratamiento. Es así como se utilizaron las categorías de clasificación de gravedad del déficit visual de la Organización Mundial de la Salud (OMS) Consultation on development of standards for characterization of vision loss and visual functioning, de 2003, donde ceguera es una agudeza visual peor que $3 / 60$ (<20/400) y baja visión se agrupa bajo dos categorías, a saber: deficiencia visual moderada y grave (agudeza visual peor que 6/18 o 20/60 hasta 3/60 20/400, respectivamente) ${ }^{14,15}$.

\section{Criterios de exclusión}

Pacientes que no tuvieran datos de comorbilidades oftalmológicas y no oftalmológicas o que hubiera incongruencia entre la agudeza visual y la certificación de discapacidad visual.

\section{Procesamiento de información}

Las variables sociodemográficas fueron edad, sexo, nivel educativo y ocupación; y las variables clínicas de interés fueron categoría de la deficiencia visual según agudeza visual, diagnóstico oftalmológico principal, comorbilidades oftalmológicas y no oftalmológicas, número de comorbilidades oftalmológicas, tabaquismo, alcoholismo y consumo de sustancias psicoactivas. La 
variable categoría de la deficiencia visual permite agrupar la población de estudio en: pacientes sin discapacidad visual (deficiencia visual sin criterio de discapacidad visual), pacientes con baja visión y pacientes con ceguera. La ceguera es una agudeza visual peor que $3 / 60(<20 / 400)$ y baja visión es una agudeza visual peor que 6/18 (20/60) hasta 3/60 (20/400).

Se identificó la etiología responsable de la deficiencia visual en cada paciente, y se agruparon de forma anatomoclínica en patologías del segmento anterior y posterior ocular, patologías mixtas (incluían ambos segmentos), patologías neurooftalmológicas y extraoculares. Las comorbilidades no oftalmológicas identificadas en los pacientes se agruparon en varias categorías: cardiovascular, endocrina, psicológica, reumatológica, neurológica, infecciosa, oncológica y traumas.

Se diseñó y utilizó una base de datos en Microsoft Excel $^{\circledR}$, que se realizó utilizando campos con validación de datos para disminuir errores en la digitación, posteriormente se verificó la calidad de la base de datos por medio de la detección de valores extremos y exploración de variables, antes de realizar el análisis.

\section{Análisis estadístico}

Para describir las variables cualitativas se calcularon frecuencias absolutas y relativas, para las variables cuantitativas, mediana con rango intercuartílico en el grupo sin discapacidad visual y media con desviación estándar en el grupo de pacientes con discapacidad. El análisis se realizó en el paquete estadístico IBM Corp. Released 2013. IBM SPSS Statistics for Windows, Version 22.0. Armonk, NY: IBM Corp.

Se calculó la razón de prevalencia (RP) con sus respectivos IC 95\% para algunas variables sociodemográficas y clínicas de interés, con el fin de evaluar si la prevalencia difiere entre grupos expuestos y no expuestos.

Para determinar si había relación, se calculó la RP entre la discapacidad visual con las siguientes variables de exposición: sexo femenino, edad superior a 50 años, tabaquismo, alcohol, sustancias psicoactivas, número de comorbilidades oftalmológicas, comorbilidades cardiovasculares, comorbilidades endocrinas, HTA y DM. A su vez, el tabaquismo se relacionó con glaucoma, cataratas y DMAE. Adicionalmente, la presencia o no de discapacidad visual fue considerada como exposición para el desarrollo de las siguientes variables: la no actividad laboral (para esta variable se tuvieron en cuenta los pacientes mayores de 18 años que reportaron ser ama de casa, desempleados, estudiantes o pensionados en la encuesta), el bajo nivel de escolaridad (definida como pacientes mayores de 18 años que no habían superado la primaria), comorbilidad endocrina y cardiovascular.

\section{Ética}

Este proyecto fue avalado por el comité de investigación y ética de la Universidad Pontificia Bolivariana.

\section{Resultados}

La población de estudio fue de 180 pacientes que hicieron parte del proyecto "Certificación de discapacidad sensorial visual: Aplicación de la Clasificación Internacional del Funcionamiento, Salud y Discapacidad (CIF-2001) en una población de 180 personas con déficit visual" y que además cumplieron con los criterios de elegibilidad de este estudio. Ninguno de los pacientes fue excluido.

La mayoría fueron mujeres, el 56.1\% (101/180), con una mediana de edad de 48 años (Q1:26.2, Q3:59.7), el $47.8 \%(86 / 180)$ de la población fue mayor de 50 años. El 79.4\% (123/180) de los pacientes no eran laboralmente activos, la ocupación más frecuente fue la de ama de casa, con el $30.6 \%$ (55/180). En los adultos activos laboralmente, se encontró una mayor proporción de hombres activos comparado con las mujeres (26/67, 36.8\% versus 6/88,6.8\%; $p<0.001)$. Esto se debe a que la mayoría de las mujeres son amas de casa $(54 \%, 54 / 100)$. El $56.1 \%(87 / 180)$ de los pacientes tenían bajo nivel educativo y el 37.8\% (68/180) habían superado la primaria. Del total de los pacientes, el $58.8 \%(106 / 180)$ tenían discapacidad visual. De estos, el 50\% (53/106) se correspondían a baja visión moderada, el $8.4 \%$ (9/106), a baja visión grave, y el $41.5 \%$ (44/106), a ceguera.

En la Tabla 1 se describen las características sociodemográficas de los 180 pacientes divididos en tres grupos de acuerdo con el grado de deficiencia visual.

La mayoría de los pacientes sin discapacidad y con baja visión eran mujeres, mientras que los pacientes con ceguera, la mayoría eran hombres. En cuanto a la ocupación, la más frecuente en los tres grupos fue la de ama de casa, los pacientes sin discapacidad tenían mayor frecuencia de no actividad laboral, 17 pacientes (23.3\%), seguido del grupo de ceguera, 5 pacientes (11.4\%), y de baja visión, 6 pacientes $(9.7 \%)$. Los pacientes que no aplicaron para la ocupación fueron: seis menores de cinco años que aún no estaban en guardería y un paciente que estaba en un hogar geriátrico. 
Tabla 1. Características sociodemográficas de los 180 pacientes atendidos en dos instituciones oftalmológicas de Medellín, 2015

\begin{tabular}{|c|c|c|c|}
\hline Característica sociodemográfica & $\begin{array}{c}\text { Sin discapacidad visual } \\
\text { n: } 74(\%)\end{array}$ & $\begin{array}{l}\text { Baja visión } \\
\text { n: } 62(\%)\end{array}$ & $\begin{array}{l}\text { Ceguera } \\
\text { n: } 44(\%)\end{array}$ \\
\hline $\begin{array}{l}\text { Sexo } \\
\text { Masculino } \\
\text { Femenino }\end{array}$ & $\begin{array}{l}32(43.2) \\
42(56.8)\end{array}$ & $\begin{array}{l}24(38.7) \\
38(61.3)\end{array}$ & $\begin{array}{l}23(52.3) \\
21(47.7)\end{array}$ \\
\hline $\begin{array}{l}\text { Ocupación } \\
\text { Ama de casa } \\
\text { Desempleado } \\
\text { Activo } \\
\text { Estudiante } \\
\text { Pensionado } \\
\text { No aplica }\end{array}$ & $\begin{array}{c}19(26) \\
17(23.3) \\
13(17.8) \\
13(17.8) \\
9(12.3) \\
2(2.7)\end{array}$ & $\begin{array}{c}22(35.5) \\
6(9.7) \\
9(14.5) \\
18(29) \\
7(11.3) \\
0(0)\end{array}$ & $\begin{array}{c}14(31.8) \\
5(11.4) \\
10(22.7) \\
7(15.9) \\
3(6.8) \\
5(11.4)\end{array}$ \\
\hline $\begin{array}{l}\text { Nivel educativo } \\
\text { Ninguno } \\
\text { Primaria } \\
\text { Secundaria } \\
\text { Técnico/tecnólogo } \\
\text { Universitario } \\
\text { Posgrado } \\
\text { No aplica }\end{array}$ & $\begin{array}{c}22(29.7) \\
19(25.7) \\
15(20.3) \\
6(8.1) \\
7(9.5) \\
3(4.1) \\
2(2.7)\end{array}$ & $\begin{array}{c}11(17.7) \\
25(40.3) \\
13(21) \\
6(9.7) \\
5(8.1) \\
2(3.2) \\
0(0)\end{array}$ & $\begin{array}{c}15(34.1) \\
16(36.4) \\
6(13.6) \\
2(4.5) \\
3(6.8) \\
0(0) \\
2(4.5)\end{array}$ \\
\hline Edad (años) & $48.5(30.75-58)^{*}$ & $46.24( \pm 22.14)^{\dagger}$ & $43.23( \pm 24.03)$ \\
\hline
\end{tabular}

*Mediana (01-03).

†Media (desviación estándar).

Para las variables correspondientes a laboralmente activo y bajo nivel educativo se tomaron en cuenta los pacientes mayores de 18 años (155 pacientes). Más del $70 \%$ de los pacientes no estaban laboralmente activos, independientemente del grupo según su capacidad visual: $83 \%$ con deficiencia visual sin criterio de discapacidad visual, $80 \%$ baja visión y $73 \%$ ceguera. Sin embargo, se encontró una frecuencia similar en los pacientes activos en los tres grupos, que fue un 27\% en el grupo de ceguera (10/37), frente al $20 \%$ en el grupo sin discapacidad (13/64) y el $19.5 \%$ en el grupo de baja visión (9/54). La mayoría de los pacientes en los tres grupos tenían bajo nivel educativo, el grupo en el que había más pacientes con esta característica fue el de ceguera, con un $70.3 \%$ (26/37), seguido del grupo de baja visión y sin discapacidad.

Respecto al diagnóstico principal responsable de la deficiencia visual, el segmento más afectado en los tres grupos fue el posterior, con el $46.7 \%$ (84/180), y las patologías más frecuentes fueron el glaucoma, la DMAE y la retinitis pigmentosa. El segundo segmento más afectado fue el anterior, con $22.8 \%$ (41/180), donde las patologías más frecuentes fueron miopía y catarata, seguido de las patologías neurooftalmológicas, con $15.6 \%(28 / 180)$, de las cuales las más frecuentes fueron la neuropatía óptica compresiva, isquémica y traumática.
El $66.1 \%$ de los pacientes (119/180) presentaron al menos una comorbilidad oftalmológica. De estos, el $41.7 \%$ (75/180) tenían una sola, el 20\% (36/180) tenían dos y el $4.4 \%$ (8/180) tenían tres o más comorbilidades. El segmento más afectado por las comorbilidades oftalmológicas fue el anterior, con el $64.7 \%$ (77/119), la comorbilidad más frecuente fue la catarata. Al discriminarlo por grupos, el segmento anterior representó el $50 \%(32 / 64)$ del grupo sin discapacidad, el $47.7 \%$ (21/44) del grupo ceguera y el $45.2 \%$ (28/62) del grupo con baja visión.

La comorbilidad no oftalmológica más frecuente fue la cardiovascular, que se presentó en el 37.7\% (68/180), seguida de la endocrina, que se presentó en un $21.1 \%$ (38/180). El tabaquismo se presentó en el $14.4 \%$ (26/180), el $3.8 \%(7 / 180)$ eran consumidores de psicotrópicos y el $24.4 \%$ (44/180) eran consumidores de alcohol. En la Tabla 2 se muestra en detalle las características clínicas encontradas en los tres grupos de pacientes del estudio.

No se encontraron asociaciones estadísticamente significativas entre la discapacidad y las variables analizadas (Tabla 3 y 4). Así mismo, al comparar la baja visión con ceguera, no se encontraron asociaciones estadísticamente significativas con las variables sexo femenino (RP: 0.7; IC 95\%: 0.4-1.1), actividad laboral (RP: 1.6; IC 95\%: 0.7-3.6), nivel educativo (RP: 1.3; IC 
Tabla 2. Características clínicas de los 180 pacientes atendidos en dos instituciones oftalmológicas de Medellín, 2015

\begin{tabular}{|c|c|c|c|}
\hline Variable & Sin discapacidad n: $74(\%)$ & Baja visión n: 62 (\%) & Ceguera n: $44(\%)$ \\
\hline Tabaquismo & $10(13.5)$ & $10(16.1)$ & $6(13.6)$ \\
\hline Consumo de alcohol & $16(21.6)$ & $16(25.8)$ & $12(27.3)$ \\
\hline Psicotrópicos & $2(2.7)$ & $5(8)$ & $0(0)$ \\
\hline $\begin{array}{l}\text { Diagnóstico principal } \\
\text { Segmento posterior } \\
\text { Segmento anterior } \\
\text { Neurooftalmológico } \\
\text { Mixto } \\
\text { Sin diagnostico } \\
\text { Extraocular }\end{array}$ & $\begin{array}{c}33(44.6) \\
12(16.2) \\
15(20.3) \\
10(13.5) \\
2(2.7) \\
2(2.7)\end{array}$ & $\begin{array}{c}30(48.4) \\
19(30.6) \\
8(12.9) \\
4(6.5) \\
1(1.6) \\
0(0)\end{array}$ & $\begin{array}{c}21(47.7) \\
10(22.7) \\
5(11.4) \\
7(15.9) \\
1(2.3) \\
0(0)\end{array}$ \\
\hline $\begin{array}{l}\text { Número diagnóstico oc } \\
0 \\
1 \\
2 \\
3\end{array}$ & $\begin{array}{c}27(36.5) \\
34(45.9) \\
11(14.9) \\
2(2.7)\end{array}$ & $\begin{array}{c}18(29) \\
25(40.3) \\
17(27.4) \\
2(3.2)\end{array}$ & $\begin{array}{c}16(36.4) \\
16(36.4) \\
8(18.2) \\
4(9.1)\end{array}$ \\
\hline $\begin{array}{l}\text { Comorbilidades no ofta } \\
\text { Cardiovascular } \\
\text { Endocrina } \\
\text { Otra discapacidad } \\
\text { Psicológica } \\
\text { Reumatológica } \\
\text { Neurológica } \\
\text { Infecciosa } \\
\text { Oncológica } \\
\text { Traumatológica } \\
\text { Otros* }\end{array}$ & $\begin{array}{c}26(35.1) \\
10(13.5) \\
11(14.9) \\
8(10.8) \\
2(2.7) \\
5(6.8) \\
5(6.8) \\
1(1.4) \\
10(13.5) \\
2(2.7)\end{array}$ & $\begin{array}{c}28(45.2) \\
18(29.1) \\
10(16.1) \\
8(12.9) \\
1(1.6) \\
3(4.8) \\
1(1.6) \\
3(4.8) \\
5(8.1)\end{array}$ & $\begin{array}{c}14(31.8) \\
10(22.7) \\
6(13.6) \\
4(9.1) \\
1(2.2) \\
5(11.4) \\
1(2.2) \\
3(6.8) \\
2(4.5)\end{array}$ \\
\hline
\end{tabular}

95\%: 0.9-1.8), tabaquismo (RP: 0.8; IC 95\%: 0.4-1.7), alcohol (RP: 1; IC 95\%: 0.6-1.7), HTA (RP: 0.9; IC 95\%: 0.9-1.8) y DM (RP: 0.2; IC 95\%: 0.04-1.8).

Para establecer la relación entre tabaquismo y catarata, glaucoma y DMAE se tomaron en cuenta solo los pacientes mayores de 18 años (155 pacientes), de los cuales, 26 pacientes eran fumadores actuales. Se encontró que de los pacientes con catarata ocho eran fumadores (RP: 0.9; IC 95\%: 0.5-1.8), de los que tenían glaucoma seis eran fumadores (RP: 1.8; IC 95\%: 0.84.3) y de aquellos con DMAE solo uno era fumador (RP: 0.4; IC 95\%: 0.05-3).

\section{Discusión}

Se encontraron frecuencias relevantes de ciertas variables en los diferentes grupos de estudio. En el grupo de pacientes con discapacidad visual, el promedio de edad fue cercano a los 50 años, predominó el sexo femenino, el bajo nivel de escolaridad, la no actividad laboral, el consumo de alcohol, tabaco y sustancias psicoactivas. Respecto a las características clínicas, se encontró que los pacientes con discapacidad visual presentaron más comorbilidades oftalmológicas y no oftalmológicas, destacándose la DM y la HTA.

En nuestro estudio, el $47.2 \%$ de los pacientes con discapacidad visual eran mayores de 50 años, lo cual es similar a los resultados de un estudio realizado en Nigeria ${ }^{16}$ en el que se caracterizó el perfil clínico y demográfico en pacientes con discapacidad visual, y se encontró que en una muestra de 193 pacientes entre los 6 y los 90 años la media de edad fue de 41.4 años y el porcentaje de pacientes mayores de 50 años fue del $42 \%$, cifras, además, relacionadas con los estudios realizados en Malasia ${ }^{17}$, Corea $^{18}$, Nepal ${ }^{19}$ e India ${ }^{20}$, donde la proporción de pacientes mayores de 50 años fue del $26,31,42$ y $32 \%$, respectivamente. En contraste, la OMS reportó que un $65 \%$ de los pacientes con discapacidad visual fueron mayores de 50 años ${ }^{1}$, similar a los resultados de un estudio en Corea del Sur en el cual se asoció la discapacidad visual con una edad mayor a 70 años ${ }^{10}$. Los resultados del presente estudio podrían estar explicados por el hecho de realizarse en un país en 
Tabla 3. Relación entre factores clínicos y sociodemográficos con la discapacidad visual de los 180 pacientes atendidos en dos instituciones oftalmológicas de Medellín, 2015

\begin{tabular}{|l|c|c|c|}
\hline Exposición & Discapacidad visual n: $106(\%)$ & Sin discapacidad visual n: $74(\%)$ & RP (IC 95\%) \\
\hline Sexo femenino & $59(55.6)$ & $42(56.7)$ & $0.9(0.7-1.2)$ \\
\hline Mayor de 50 años & $50(47.2)$ & $36(48.6)$ & $0.9(0.7-1.2)$ \\
\hline Bajo nivel educativo & $54(50.9)$ & $33(44.6)$ & $1.1(0.8-1.5)$ \\
\hline No actividad laboral & $72(67.9)$ & $51(68.9)$ & $0.9(0.8-1.2)$ \\
\hline Al menos una comorbilidad oftalmológica & $72(67.9)$ & $47(63.51)$ & $1.0(0.8-1.4)$ \\
\hline Comorbilidades cardiovasculares & $42(39.6)$ & $26(35.1)$ & $1.1(0.7-1.6)$ \\
\hline Hipertensión & $36(33.9)$ & $16(21.6)$ & $1.2(0.9-1.6)$ \\
\hline Comorbilidades endocrinológicas & $21(19.8)$ & $10(13.5)$ & $1.4(0.7-2.9)$ \\
\hline Diabetes mellitus & $8(7.5)$ & $3(4)$ & $1.2(0.8-1.8)$ \\
\hline Tabaquismo & $16(15)$ & $10(13.5)$ & $1.0(0.7-1.4)$ \\
\hline Alcohol & $28(23.6)$ & $16(21.6)$ & $1.1(0.8-1.4)$ \\
\hline Sustancias psicoactivas & $5(4.71)$ & $2(2.7)$ & $1.2(0.7-1.9)$ \\
\hline
\end{tabular}

Tabla 4. Relación entre comorbilidades no oftalmológicas y discapacidad visual

\begin{tabular}{|l|c|c|c|}
\hline Comorbilidad & Con discapacidad n: $\mathbf{1 0 6}(\%)$ & Sin discapacidad n: $74(\%)$ & RP (IC 95\%) \\
\hline Infecciosa & 7 & 5 & $0.9(0.6-1.6)$ \\
\hline Reumatológica & 2 & 2 & $0.8(0.3-2.2)$ \\
\hline Neurológica & 8 & 5 & $1.0(0.6-1.6)$ \\
\hline Oncológica & 2 & 1 & $1.1(0.5-2.5)$ \\
\hline Psicológica & 11 & 8 & $0.9(0.4-2.2)$ \\
\hline Traumatológica & 6 & 10 & $0.6(0.3-1.1)$ \\
\hline Otra discapacidad & 15 & 11 & $0.9(0.6-1.3)$ \\
\hline Otra comorbilidad & 7 & 2 & $1.3(0.9-1.9)$
\end{tabular}

vía de desarrollo, en el cual la expectativa de vida es menor en comparación con los países desarrollados ${ }^{21}$.

Respecto al sexo, se encontró que el $55.6 \%$ de la población con discapacidad visual era femenina, resultado que concuerda con otros estudios que reportan que el $60 \%$ de la población con ceguera son mujeres ${ }^{21}$. Los trastornos de la visión afectan con mayor frecuencia a las mujeres en comparación con los hombres. Este fenómeno se hace más evidente en países de altos ingresos cuando se trata de discapacidad visual tipo ceguera ${ }^{22}$. Adicionalmente se encontró que en los pacientes con discapacidad visual la ocupación más frecuente fue la de ama de casa, distribuida en 35.5\% para baja visión y $31.8 \%$ para ceguera. Estos resultados pueden estar sujetos a que esta ocupación es generalmente asumida por mujeres. El informe mundial de discapacidad de la $\mathrm{OMS}^{3}$ indica una leve inclinación de la discapacidad hacia el sexo femenino y, además, se reporta que las mujeres tienen menor acceso a los servicios de salud, un hecho que puede estar explicado por sus bajos ingresos económicos, mayor carga económica en la atención sanitaria y por su rol en el cuidado de la familia y ámbito doméstico que limitan el tiempo para el acceso a la atención médica ${ }^{23}$.

Se observó que los pacientes con discapacidad visual tenían menor nivel educativo, pues si bien no hay muchos estudios que lo soportan, se conoce que el $87 \%$ de las personas con discapacidad visual habitan países en desarrollo, lo cual deriva en un nivel de educación bajo y un acceso reducido a los servicios de 
sanitaros, lo que impacta negativamente en el cuidado de la salud con un probable aumento de discapacidad visual por causas infecciosas o una menor motivación a la asistencia médica precoz ${ }^{21}$.

Con relación al diagnóstico principal que originó la deficiencia visual permanente, el segmento posterior fue el más comprometido, pues del total de la población, el 46.7\% padecía glaucoma, y este produce una neuropatía óptica glaucomatosa que altera gravemente la visión, seguido del segmento anterior, donde la enfermedad más frecuente fue la miopía, que afectó al $22.8 \%$, y el eje neurooftalmológico, que afectó al $15.6 \%$. Sin embargo, según reportes de la OMS del año 2018, las causas globales de discapacidad visual son las alteraciones del segmento anterior, como defectos refractivos no corregidos $(53 \%)$ y la catarata $(25 \%)$, mientras que el glaucoma es responsable solo del $8 \%{ }^{6}$. Si bien en Colombia, México y Brasil la ametropía más frecuente que se relaciona con baja visión y ceguera es la hipermetropía ${ }^{24}$, en este estudio fue la miopía.

De las comorbilidades no oftalmológicas, se sabe que la retinopatía diabética explica alrededor del 5\% de la ceguera en el mundo como una de las complicaciones microvasculares, al igual que la HTA, la cual se ha considerado como un factor importante para el desarrollo de retinopatía. Se ha descrito, además, que cuando estas dos patologías se asocian, el riesgo se eleva considerablemente ${ }^{25}$. En este estudio, la enfermedad cardiovascular y la endocrina fueron las comorbilidades no oftalmológicas más frecuentes, sin embargo, ambas asociaciones con la discapacidad visual no arrojaron valores estadísticamente significativos.

En relación con el consumo de sustancias, se encontró que el consumo de alcohol era más frecuente en la población con discapacidad visual que en la población sin dicha condición (23.6 vs. $21.6 \%$ ), al igual que el tabaquismo (15 vs. $13.5 \%$ ). Si bien se ha demostrado que el consumo de estas sustancias afecta estructuras oculares, en el caso del tabaco por componentes como el cianuro, y en el del alcoholismo por deficiencias de vitamina B12 y folatos, lo que aumenta el riesgo de neuropatía óptica ${ }^{8}$, la relación de consumo de estas sustancias y discapacidad visual no fue estadísticamente significativa, probablemente porque la cantidad de personas que consumía dichas sustancias era muy baja en ambas poblaciones.

El presente estudio es un primer acercamiento analítico para identificar y postular relaciones entre algunos factores sociodemográficos y clínicos con la discapacidad categoría sensorial tipo visual. Tiene relevancia científica por ser un estudio pionero en Colombia, a través de una metodología observacional, de corte transversal y analítica, en una población que cumple con criterios clínicos de baja visión y ceguera. Sin embargo, tiene una serie de limitaciones, las cuales deben ser tenidas en cuenta: a) tamaño reducido de la población, razón por la cual las relaciones no fueron estadísticamente significativas; y b) sesgo de selección, porque las personas con discapacidad visual fueron obtenidas a conveniencia de dos centros oftalmológicos de referencia en discapacidad visual de la ciudad de Medellín, y no de la población en general, aspecto que limita la validez externa de los resultados.

Los resultados obtenidos permiten un acercamiento a la realidad local en la ciudad de Medellín, e identificar mejor algunas características clínicas y sociodemográficas (poco reportadas en la literatura) sobre un reto en salud visual como lo es la discapacidad visual (baja visión y ceguera), y brindar información aplicable para el personal de salud que atiende población con discapacidad, siendo esto un paso importante en el mejoramiento de su calidad de vida y atención en salud, y primer paso para proponer otros estudios observacionales y de intervención en el tema. Este estudio tendrá una siguiente fase a través de la investigación «Etiología de baja visión y ceguera en diferentes instituciones especializadas en oftalmología o con servicios de oftalmología de referencia en Colombia», estudio transversal multicéntrico en seis ciudades (estudio finalizado en enero del año 2018).

\section{Conclusión}

Se encontraron frecuencias relevantes de ciertas variables en los diferentes grupos de estudio que sugieren que hay algún tipo de relación con la discapacidad, ya sea como factor de riesgo o como desenlace. Es necesario continuar realizando estudios que combinen aspectos sociodemográficos con variables clínicas en pro de redireccionar acciones de promoción, prevención y rehabilitación.

\section{Responsabilidades éticas}

Protección de personas y animales. Los autores declaran que los procedimientos seguidos se conformaron a las normas éticas del comité de experimentación humana responsable y de acuerdo con la Asociación Médica Mundial y la Declaración de Helsinki.

Confidencialidad de los datos. Los autores declaran que han seguido los protocolos de su centro de trabajo sobre la publicación de datos de pacientes.

Derecho a la privacidad y consentimiento informado. Los autores han obtenido el consentimiento 
informado de los pacientes y/o sujetos referidos en el artículo. Este documento obra en poder del autor de correspondencia.

\section{Conflicto de intereses}

\section{Los autores declaran que no tienen conflicto de intereses.}

\section{Bibliografía}

1. Who.int [Internet]. Ginebra: OMS; 2014 [actualizado 16 de enero de 2018; citado 22 de junio de 2018]. Disponible en: http://www.who.int/es/ news-room/fact-sheets/detail/disability-and-health.

2. García Ramos CE. Guía de atención educativa para estudiantes con discapacidad visual. [Internet]. Aguascalientes: Instituto de Educación de Aguascalientes; 2012 [Citado 22 de junio de 2018]. Disponible en: http:// www.sld.cu/galerias/pdf/sitios/histologia/normas-vancouver-buma-2013-guia-breve.pdf.

3. OMS, Banco Mundial. Informe Mundial sobre la Discapacidad [Internet] Malta: OMS, BM; 2011. Disponible en: http://www.who.int/disabilities/ world report/2011/summary es.pdf.

4. Stevens GA, White RA, Flaxman SR, Price H, Jonas JB, Keeffe J, et al. Global prevalence of vision impairment and blindness: Magnitude and temporal trends, 1990-2010. Ophthalmology. 2013;120(12):2377-84.

5. Bourne RRA, Flaxman SR, Braithwaite T, Cicinelli MV, Das A, Jonas JB, et al. Vision Loss Expert Group. Magnitude, temporal trends, and projections of the global prevalence of blindness and distance and near vision impairment: a systematic review and meta-analysis. Lancet Glob Health. 2017; 5 (9): e888-e897.

6. Who.int [Internet]. Ginebra: OMS; agosto de 2014 [actualizado 11 octubre de 2018; citado junio 26 de 2018]. Disponible en: http://www.who.int/es/ news-room/fact-sheets/detail/blindness-and-visual-impairment.

7. Who.int [Internet]. Ginebra: OMS; agosto de 2014 [citado junio 26 de 2018]. Disponible en: https://www.who.int/features/factfiles/blindness/es/.

8. Rojas S, Ruíz S, Carvajal J, Álvarez MJ, Duque D, Correa SA, et al. Caracterización de una población con discapacidad visual (baja visión y ceguera) atendida en dos Instituciones Prestadoras de Salud de Medellín. Med U.P.B. 2015;34(1):30-9.

9. Beltran JC, González C; Fundación Saldarriaga Concha, Colombia Líder. Discapacidad en Colombia: Reto para la Inclusión en Capital Humano. [Internet]. Tomo I. Bogotá: Fundación Saldarriaga Concha; abril 2010 [citado julio 1 de 2018]. Disponible en: https://www.saldarriagaconcha. org/desarrollo fsc/images/fsc/pdf/biblioteca virtual/discapacidad/estadisticas_e_investigaciones/05_tomo_1_exclusion_DEPTAL_CH.pdf.

10. Park SH, Lee JS, Heo H, Suh YW, Kim SH, Lim KH, et al. Epidemiologic Survey Committee in the Korean Ophthalmological Society. A nationwide population-based study of low vision and blindness in South Korea. InvestOphthalmol Vis Sci. 2014 De;56(1):484-93.

11. Zhang X, Kahende J, Fan AZ, Barker L, Thompson TJ, Mokdad AH, Li Y, Saaddine JB. Smoking and visual impairment among older adults with age-related eye diseases. Prev Chronic Dis. 2011;8(4):A84.

12. Velazco V. Discapacidad y Salud. El consumo de drogas y sus secuelas [Internet]. Argentina, enero 2011 [citado julio 3 2018]. Disponible en: http://discapacidadrosario.blogspot.com/2011/01/el-consumo-de-drogasy-sus-secuelas.html.

13. Organización Mundial de la Salud. Neurociencia del consumo y dependencia de sustancias psicoactivas. Ginebra. p. 23. [citado julio 3 de 2018]. Disponible en: http://www.who.int/substance_abuse/publications/ en/Neuroscience_S.pdf.

14. Bourne R, Price H, Taylor $\mathrm{H}$, Leasher J, Keeffe J, Glanville J, et al. New systematic review methodology for visual impairment and blindness for the 2010 Global Burden of Disease study. Ophthalmic Epidemiol. 2013;20(1):33-9.

15. Bourne RRA, Flaxman SR, Braithwaite T, Cicinelli MV, Das A, Jonas JB, et al. Magnitude, temporal trends, and projections of the global prevalence of blindness and distance and near vision impairment: a systematic review and meta-analysis. Lancet Glob Health. 2017:5(9):e888-97.

16. Olusanya B, Onoja G, Ibraheem W, Bekibele C. Profile of patients presenting at a low vision clinic in a developing country. BMC Ophthalmol. 2012:30:12:31.

17. Mohidin N, Yusoff S. Profile of a low vision clinic population. Clin Exp Optom. 1998;81(5):198-202.

18. Kim JH, Joo KS, Moon NJ. Characteristics of 681 low vision patients in Korea. J Korean Med Sci. 2010;25(8):1217-21.

19. Paudel P, Khadka J, Sharma AK. Profile of a low vision population. Int Congr Ser. 2005;1282:252-6.

20. Khan SA. A retrospective study of low-vision cases in an Indian tertiary eye-care hospital. Indian J Ophthalmol. 2000;48(3):201-7.

21. Discapacidad visual y ceguera en el adulto: Revisión de tema. Juan Camilo Suárez Escudero. MEDICINA U.P.B. 2011;30(2):170-80.

22. Bourne RRA, Flaxman SR, Braithwaite T, Cicinelli MV, Das A, Jonas JB, et al. Magnitude, temporal trends, and projections of the global prevalence of blindness and distance and near vision impairment: a systematic review and meta-analysis. Lancet Glob Health. 2017;5(9):e888-97.

23. UNESCO. Informe de la salud visual en Suramérica 2008: cátedra Unesco Salud Visual y Desarrollo. Madrid: Unesco; 2008.

24. Roselló Leyva A, Rodríguez Masó S, Rojas Rondón I, Linares Guerra M Ramos Gómez EA, Vázquez Adan Yanis. Defectos refractivos más frecuentes que causan baja visión. Rev Cubana Oftalmol. 2011;24(2):271-8.

25. Gallego PH, Craig ME, Hing S, Donaghue KC. Role of blood pressure in development of early retinopathy in adolescents with type 1 diabetes: prospective cohort study. BMJ. 2008;26;337:a918. 